\title{
Investigating the impact of dietary habits, sun exposure, and body composition on serum $250 H D$ concentrations at five years of age - Findings from the ROLO Kids Study
}

\author{
M.K. McVey ${ }^{1}$, A.A. Geraghty ${ }^{1}$, E.C. O’Brien ${ }^{1}$, M.J. McKenna ${ }^{1,2,3}$, M.T. Kilbane ${ }^{4}$, \\ R.K. Crowley, ${ }^{2,}$, P.J. Twomey ${ }^{3,4}$ and F.M. McAuliffe ${ }^{1}$ \\ ${ }^{1}$ UCD Perinatal Research Centre, Obstetrics \& Gynaecology, School of Medicine, National Maternity Hospital, \\ University College Dublin, Dublin, Ireland, ${ }^{2}$ Department of Endocrinology, St. Vincent's University Hospital, Dublin, \\ Ireland, ${ }^{3}$ School of Medicine, University College Dublin, Dublin, Ireland and ${ }^{4}$ Department of Clinical Chemistry, \\ St. Vincent's University Hospital, Dublin, Ireland.
}

Serum 25-hydroxyvitamin D (25OHD) is the main circulating form of vitamin D in the blood. Recent research suggests that inadequate vitamin D status is common in early childhood ${ }^{(1)}$. Vitamin D status in adults is determined by numerous factors such as oral intake, sun exposure, and body composition. Research is limited regarding determinants of 25OHD status in young children.

Analysis was carried out on 79 children from the ROLO Kids study in Dublin, Ireland. Blood samples were collected for serum 25OHD estimation. Dietary intakes and dietary habits were measured using a food frequency questionnaire and lifestyle questionnaire completed by the mother. Child weight, height, and skinfolds were measured by trained researchers. Statistical analysis included Spearman correlations and Mann-Whitney U tests.

$61 \%$ of the participants had sufficient $25 \mathrm{OHD}(\geq 50 \mathrm{nmol} / \mathrm{L})$, using the $2011 \mathrm{IOM}$ report on dietary reference intakes for calcium and vitamin $\mathrm{D}$ as the basis for interpretation ${ }^{(2)}$. Neither reported dietary vitamin $\mathrm{D}$ nor calcium intake was significantly associated with 25OHD. Intakes of standard milk, eggs, and oily fish were not associated with 25OHD, but the consumption of fortified milk, and more than 7 bowls of cereal a week were independently associated with higher 25OHD ( $<<0 \cdot 001$ and $p=0 \cdot 049$, respectively). There was no association of body composition at 5 years with 25OHD. Sun exposure (measured as obtaining at least half an hour of sun per day) was not significantly associated with $25 \mathrm{OHD}$, but reported use of sunscreen was associated with increased $25 \mathrm{OHD}$ $(\mathrm{p}=0 \cdot 016)$.

Table 1. Comparing dietary habits and the association with serum 25OHD concentrations in 5-year old children.

\begin{tabular}{llcc}
\hline Dietary Habits & N & Median (IQR) (nmol/L) & P \\
\hline$\geq 200$ mls of Milk/day & 50 & $53 \cdot 5(28 \cdot 25)$ & $0 \cdot 885$ \\
$<200 \mathrm{mls}$ of Milk/day & 13 & $52(30 \cdot 5)$ & \\
Consumed Supermilk & 10 & $84.5(68)$ & $\mathbf{0 . 0 0 1 *}$ \\
Did not consume Supermilk & 63 & $51(30)$ & \\
$\geq 1$ egg/week & 53 & $56(27)$ & $0 \cdot 272$ \\
$<1$ egg/week & 13 & $50 \cdot 5(32 \cdot 25)$ & $0 \cdot 767$ \\
Any oily fish intake & 43 & $55(27)$ & \\
No oily fish intake & 27 & $52(44)$ & $\mathbf{0 . 0 4 9 *}$ \\
$\geq 7$ or more bowls of cereal/week & 42 & $62.5(28)$ & \\
$<7$ bowls of cereal a week & 29 & $47(21)$ & $\mathbf{0 . 0 1 6 *}$ \\
Lifestyle Habits & & $59(25)$ & \\
Always/Usually use Sunscreen & 43 & $49(39)$ & \\
Rarely/Never use Sunscreen & 29 &
\end{tabular}

$\mathrm{P}$ values were calculated using Mann Whitney-U tests.

*P-value is significant at $<0 \cdot 05$.

These findings suggest the primacy of dietary and lifestyle habits as determinants of 25OHD in early childhood. Further education surrounding dietary habits may be useful to ensure sufficient vitamin D status within this age group.

1. Ward L, Ladhani M et al. (2017) Pediatric Child Health 22(1), 43-44.

2. Ross A, Manson J et al. (2011) J Clin Endocrinol Metab 96(1), 53-58. 